\section{The advantage of sex in evolving yeast populations}

\section{Clifford Zeyl $\star_{\dagger}$ \& Graham Bell $\ddagger$}

* Center for Microbial Ecology, Michigan State University, East Lansing, Michigan 48824, USA

$\ddagger$ Department of Biology and Redpath Museum, McGill University, Montréal, Québec H3A 1B1, Canada

Sex is a general feature of the life cycle of eukaryotes. It is not universal, however, as many organisms seem to lack sex entirely ${ }^{1}$. The widespread occurrence of sex is puzzling, both because meiotic recombination can disrupt co-adapted combinations of genes, and because it halves the potential rate of reproduction in organisms with strongly differentiated male and female gametes ${ }^{2}$. Most attempts to explain the maintenance of sexuality invoke differences between parents and sexual offspring. These differences may be advantageous in novel or changing environments if new gene combinations are favoured from time to time ${ }^{1}$. Sex would then serve to concentrate beneficial mutations that have arisen independently into the same line of descent. But in a stable environment sex might serve to concentrate deleterious mutations, so that they will be more effectively purged from the population by selection ${ }^{3}$. We have studied the effect of sex on mean fitness in experimental populations of the budding yeast Saccharomyces cerevisiae. Our results show that sex increases mean fitness in an environment to which the populations were well adapted, but not in an environment to which new adaptation occurred, supporting the hypothesis that the advantage of sexuality lay in the removal of deleterious mutations.

Sex is thought either to accelerate adaptation to a changing environment, or to retard the loss of adaptation in a stable environment. These are not mutually exclusive theories ${ }^{4}$, and it has also been suggested that sex can facilitate the establishment of beneficial mutations by disengaging them from linked deleterious mutations ${ }^{5}$. Although these theories have been investigated through models and simulations, few experiments have been designed to test them. The hypothesis that sex accelerates adaptation to a changing environment is supported by the results of two experiments in which recombination rates were manipulated: bacteriophage T4 populations with high recombination rates evolved proflavine resistance more rapidly than did low-recombination populations ${ }^{6}$, and suppression of recombination slowed the response to artificial selection on sternopleural bristle numbers in experimental populations of the fruitfly Drosophila melanogaster ${ }^{7}$.

We have studied the effect of sex in S. cerevisiae. Haploid yeast exist as one of two mating types a and $\alpha$, which mate on contact with each other to form $\mathbf{a} / \alpha$ diploids. Wild-type haploids are homothallic, but the $H O$ gene required for mating-type switching in daughter cells can be inactivated, and most laboratory strains are heterothallic. When starved of nitrogen and grown on non-fermentable carbon sources such as acetate, diploids undergo meiosis and sporulation to produce haploid ascospores. The walls of ascospores are resistant to treatments such as enzymatic digestion that destroy vegetative cells. Standard laboratory techniques allow the construction of yeast genotypes that are identical except for mating type or ploidy ${ }^{8}$.

It has been shown that heterozygotes at the mating type (MAT) locus enjoy a competitive advantage over homozygotes ${ }^{9}$. A single episode of meiosis followed by syngamy between sibling spores (automixis or inbreeding) increased this advantage in highly

$\dagger$ Present address: Department of Biology, Wake Forest University, P.O. Box 7325, Winston-Salem, North Carolina 27109, USA.

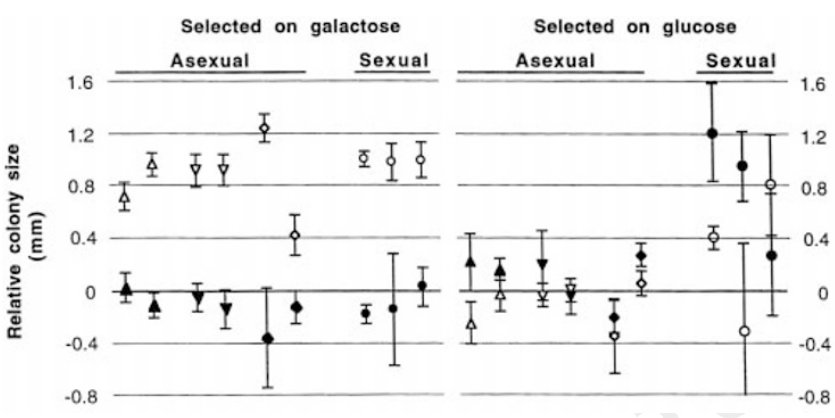

Figure 1 Growth rates of experimental yeast populations maintained on galactose or on glucose for $\sim 400-600$ generations. Mean colony sizes after three days' growth relative to a control genotype were determined for ten genotypes from each population both on the medium on which they were maintained (white symbols) and on the other medium (black symbols). Plotted values are means of the ten genotype means, with 95\% confidence intervals, for replicate haploid mating type a $(\Delta)$, haploid mating type $\alpha(\nabla)$, diploid $(\diamond)$ and sexual (O) populations.

heterozygous genotypes, but initially reduced the advantage of MAT heterozygosity in otherwise homozygous genotypes. But the implications of these results for an advantage of sex are not straightforward, because MAT heterozygosity regulates the expression of many yeast genes ${ }^{10}$ and because mitotic recombination is relatively frequent in diploid yeast ${ }^{11}$.

We constructed replicate haploid mating type a, haploid mating type $\alpha$, diploid populations (all asexual), and sexual populations. The constituent genotypes were constructed as isogenic strains, so that the starting populations were not only isogenic but almost homogeneous (see Methods). Replicate experimental populations were propagated by serial transfer on one of two media: $1 \%$ yeast extract, $2 \%$ peptone and $2 \%$ glucose, or an identical medium with $2 \%$ galactose substituted for glucose. Most laboratory yeast strains are maintained on glucose, and the genotypes from which our base populations were constructed divide roughly every $90 \mathrm{~min}$ on glucose at $25^{\circ} \mathrm{C}$. They grow only about half as fast on galactose, and induce the biochemical pathways of galactose metabolism inefficiently ${ }^{12}$. Our starting populations were thus well adapted to glucose and relatively poorly adapted to galactose.

The sexual populations underwent eight sexual cycles, each separated by about 48 or 96 mitotic divisions for populations maintained on galactose and glucose, respectively, whereas the asexual populations experienced 8 parallel asexual cycles and were refrigerated during the 5-day period in each cycle when sexual populations were sporulating. Because mitotic reproduction occurred neither on the starvation medium used to induce meiosis nor during refrigeration, the environmental differences experienced by sexual and asexual populations have no selective effects. However, although we attempted to maintain similar population sizes in all treatments by transferring similar numbers of cells $\left(10^{5}\right.$ to $\left.10^{6}\right)$ at each cycle, it is possible that in some cycles the sporulation treatment resulted in smaller sexual than asexual populations.

After eight experimental cycles, vegetative fitnesses were estimated by measuring the growth rates on glucose and galactose plates of ten genotypes from each population, relative to the genotype used to construct the starting populations (which had been stored frozen in $15 \%$ glycerol).

All populations maintained on galactose adapted significantly to galactose (Fig. 1). This adaptation was specific to the galactose medium, as no such increase was seen in their growth rates when tested on glucose. We observed no differences between haploid and diploid or between sexual and asexual populations in their adaptation to galactose (Table 1). 
Table 1 ANOVA of relative colony diameters of populations maintained on galactose

\begin{tabular}{|c|c|c|c|c|}
\hline Source & d.f. & $\begin{array}{c}\text { ayed on } \\
\text { MS }\end{array}$ & Expected MS & $F$ \\
\hline $\begin{array}{l}\text { Sex } \\
\text { Replicate (sex) } \\
\text { Genotype (replicate (sex)) } \\
\text { Error }\end{array}$ & $\begin{array}{c}1 \\
7 \\
79 \\
399\end{array}$ & $\begin{array}{l}3.518 \\
3.387 \\
0.197 \\
0.020\end{array}$ & $\begin{array}{c}s_{e}^{2}+6.8 s_{\mathrm{g}(\mathrm{sr})}^{2}+64.8 s_{\mathrm{rs})}^{2}+Q_{\mathrm{s}} \\
s_{e}^{2}+5.7 s_{\mathrm{g}(\mathrm{sr})}^{2}+51.3 s_{\mathrm{r}(\mathrm{s})}^{2} \\
s_{e}^{2}+5.5 s_{\mathrm{g}(\mathrm{sr})}^{2} \\
s_{e}^{2}\end{array}$ & $\begin{array}{l}1.04 \\
17.19^{* *} \\
9.85^{* * *}\end{array}$ \\
\hline \multicolumn{5}{|c|}{ Assayed on glucose } \\
\hline $\begin{array}{l}\text { Sex } \\
\text { Replicate (sex) } \\
\text { Genotype (replicate (sex)) } \\
\text { Error }\end{array}$ & $\begin{array}{c}1 \\
7 \\
79 \\
512\end{array}$ & $\begin{array}{l}0.629 \\
0.623 \\
0.243 \\
0.028\end{array}$ & $\begin{array}{c}s_{e}^{2}+7.2 s_{\mathrm{g}(\mathrm{s})}^{2}+68.8 s_{\mathrm{r}(\mathrm{s})}^{2}+Q_{\mathrm{s}} \\
s_{e}^{2}+7.0 s_{\mathrm{g}(\mathrm{s})}^{2}+66.2 s_{\mathrm{r}(\mathrm{s})}^{2} \\
s_{e}^{2}+6.8 s_{\mathrm{g}(\mathrm{sr})}^{2} \\
s_{e}^{2}\end{array}$ & $\begin{array}{l}1.01 \\
2.56^{*} \\
8.68^{* * *}\end{array}$ \\
\hline
\end{tabular}

ANOVA of the relative colony diameters of populations maintained on galactose, with the occurrence or absence of sex as a fixed effect and replicate population and genotype as random nested effects. Among haploid populations maintained on galactose, we found no effect of mating type on growth rates on either galactose $(P>0.3)$ or glucose $(P>0.5)$. Among haploids maintained on glucose, we detected no effect of mating type on growth rates, either on glucose $(P>0.3)$ or on galactose $(P>0.4)$. Among asexual populations maintained on galactose, no effect of ploidy was detected ( $P>0.7$ on galactose, $P>0.3$ on glucose). The same was true among asexual populations maintained on glucose ( $P>0.8$ on glucose, $P>0.1$ on galactose). Both replicates of the haploid mating type a, haploid mating type $\alpha$, and diploid treatments were therefore pooled as replicates of an asexual treatment. Values of $F$ associated with probabilities of less than $0.05,0.01$ and 0.001 are marked with one, two or three asterisks, respectively. To compare genetic variances in sexual and asexual populations, we performed separate ANOVAs on sexual and asexual treatments maintained on each medium, with assay environment as a fixed effect and replicate population within assay environment, genotype within population, and replicate colony within genotype as nested random effects. To test for differences in genetic variance between sexual and asexual populations, we calculated $F$ ratios using the estimates of variance attributable to genotype from each treatment. Among populations maintained on galactose, genetic variance was somewhat greater within asexual than sexual populations $\left(F_{36.53}=1.83 ; 0.06>P>0.02\right)$, but among populations maintained on glucose, genetic variance was greater within sexual populations $\left(F_{30.36}=2.453 ; P>0.01\right)$. MS, mean

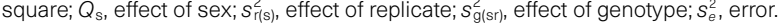

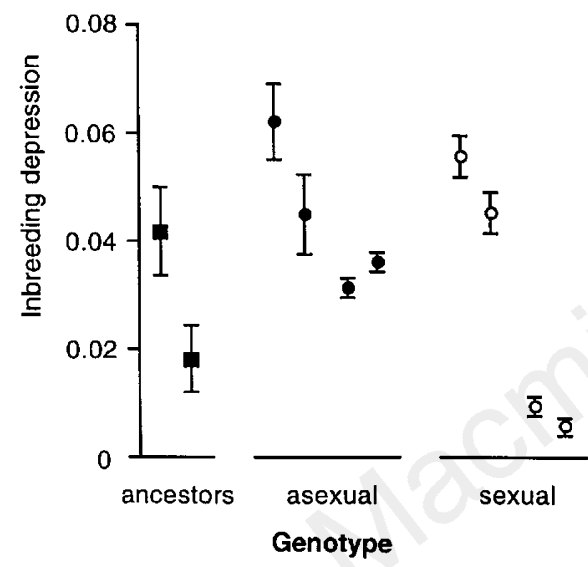

Figure 2 Inbreeding depression in ancestral genotypes and their sexual and asexual descendants. Plotted values are means and 95\% confidence intervals determined as described in Methods. For both sexual and asexual genotypes, the first two values are for genotypes are from replicate population 1 , and the third and fourth are from replicate population 2. ANOVA with life cycle (sexual or asexual) as a fixed effect and population (replicate 1 or 2 ) as a random effect nested within life cycle indicated no significant effect of life cycle $(P=0.398)$, owing to the large error term (variance attributable to the effect of replicate population). Means (and 95\% confidence intervals) for inbreeding depression are $0.027(0.016-0.038)$ for the ancestors, $0.043(0.034-0.052)$ for the asexuals and $0.029(0.013-0.045)$ for the sexuals, when the results for each replicate of each genotype are pooled.
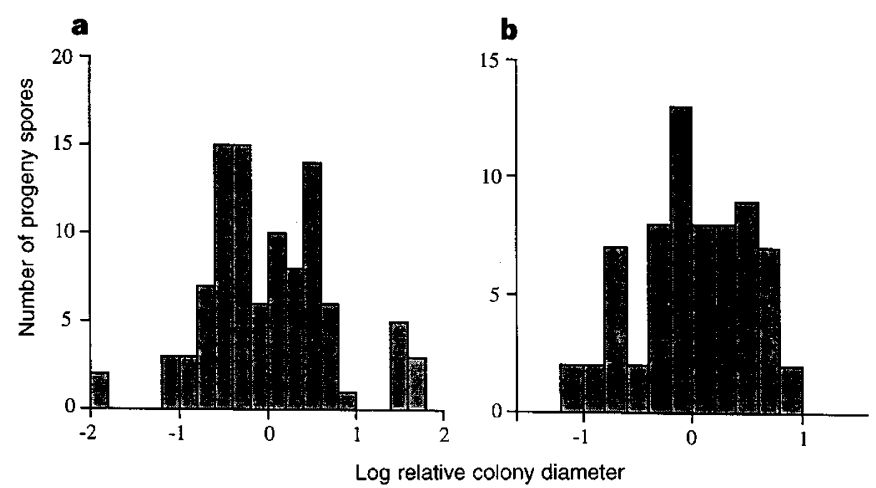

Figure 3 Frequency distributions of colony growth rates on galactose for $F_{1}$ progeny of crosses between genotypes sampled from populations that evolved on galactose, and their ancestors.
We detected no change in the performance on either medium of most of the asexual populations maintained on glucose; one diploid population grew more quickly when tested on galactose, the other diploid population grew more slowly on both media, and one of the haploid, mating type a populations grew more slowly when tested on glucose (Fig. 1). We found no effect of the test medium on the performance of any of the populations maintained on glucose, relative to the unselected control (Table 2). Thus, in contrast to the populations that evolved on galactose, those maintained on glucose acquired no detectable new adaptation to their culture medium. However, the sexual populations grew more quickly on both media than did the asexual populations (Fig. 1 and Table 2).

These two observations suggest that the superiority of the sexual populations resulted from the removal of mutations that were deleterious in both environments, rather than from the assortment of adaptive mutations into one recombinant genotype. To test this explanation, we chose two haploid genotypes from each of two sexual and two asexual haploid populations maintained on glucose, and compared the effects of inbreeding and outcrossing on the fitness of each. If the asexual genotypes carried greater numbers of deleterious mutations, and those deleterious mutations were at least partially recessive, then the fitness disadvantage of inbred versus outcrossed diploid progeny (inbreeding depression) should be greater in asexual than in sexual genotypes. We would expect any such effect to be slight, because all deleterious mutations were exposed to selection throughout the experiment in the asexual haploid population, and during the haploid phase of the sexual cycle in the sexual populations. Our results, although statistically inconclusive, are consistent with the expectation of more abundant deleterious mutations in the asexual genotypes (Fig. 2). The two ancestors of our populations carry differing and substantial loads of deleterious mutations, and the load appears to have increased in some asexual genotypes.

The maintenance of adaptation by the removal of deleterious mutations from sexual populations could occur through either of two mechanisms. First, in small asexual populations mean fitness is liable to decrease irreversibly because of the stochastic loss of the few individuals bearing the fewest mutations (Muller's ratchet ${ }^{13,14}$ ). This does not apply to sexual populations, in which genotypes with few mutations are recreated by recombination. This might be the case, for example, in yeast strains perpetuated by the repeated transfer of single colonies. Second, the mean fitness of sexual populations may exceed that of asexual populations if each additional mutation causes a greater loss of fitness in genomes that already carry greater numbers of deleterious mutations (synergistic epistasis ${ }^{3}$ ). Such synergistic interactions appear to occur between ultraviolet- 


\begin{tabular}{|c|c|c|c|c|}
\hline Source & d.f. & $\begin{array}{c}\text { sayed or } \\
\text { MS }\end{array}$ & Expected MS & $F$ \\
\hline $\begin{array}{l}\text { Sex } \\
\text { Replicate (sex) } \\
\text { Genotype (replicate (sex)) } \\
\text { Error }\end{array}$ & $\begin{array}{c}1 \\
7 \\
78 \\
502\end{array}$ & $\begin{array}{l}38.919 \\
2.012 \\
0.512 \\
0.035\end{array}$ & $\begin{array}{c}s_{e}^{2}+6.8 s_{g(s)}^{2}+62.4 s_{\mathrm{r}(\mathrm{s})}^{2}+Q_{\mathrm{s}} \\
s_{e}^{2}+7.0 s_{g(\mathrm{~s})}^{2}+65.7 s_{\mathrm{r}(\mathrm{s})}^{2} \\
s_{e}^{2}+6.7 s_{\mathrm{g}(\mathrm{sr})}^{2} \\
s_{e}^{2}\end{array}$ & $\begin{array}{l}19.34^{* *} \\
3.93^{\star *} \\
14.63^{* * *}\end{array}$ \\
\hline \multicolumn{4}{|c|}{ Assayed on galactose } & (1)..... \\
\hline $\begin{array}{l}\text { Sex } \\
\text { Replicate (sex) } \\
\text { Genotype (replicate (sex)) } \\
\text { Error }\end{array}$ & $\begin{array}{c}1 \\
7 \\
79 \\
434\end{array}$ & $\begin{array}{l}60.978 \\
8.356 \\
0.399 \\
0.044\end{array}$ & $\begin{array}{c}s_{e}^{2}+6.7 s_{\mathrm{g}(\mathrm{sr})}^{2}+59.0 s_{\mathrm{r}(\mathrm{s})}^{2}+Q_{\mathrm{s}} \\
s_{e}^{2}+6.4 s_{\mathrm{g}(\mathrm{sr})}^{2}+57.5 s_{\mathrm{r}(\mathrm{s})}^{2} \\
s_{e}^{2}+5.9 s_{\mathrm{g}(\mathrm{s})}^{2} \\
s_{e}^{2}\end{array}$ & $\begin{array}{c}7.30^{*} \\
20.94^{* * *} \\
9.07^{* * *}\end{array}$ \\
\hline
\end{tabular}

ANOVA of the relative colony diameters of populations maintained on glucose, as in Table 1. ANOVA of the populations maintained on glucose, with assay medium glucose or galactose as a fixed effect, and replicate population within assay medium and genotype within replicate population as random effects, revealed no effect on relative colony diameter of the assay medium $(P>0.1)$.

induced and naturally accumulated deleterious mutations in Chlamydomonas moewusii ${ }^{15}$. We cannot distinguish between these possibilities from our results.

Although recombination would cause the same redistribution among genomes of deleterious mutations in sexual populations on galactose as in sexuals on glucose, the former showed no fitness advantage over their asexual counterparts. The increase in growth rates on galactose of all populations maintained on galactose greatly exceeded the effect of sex among populations maintained on glucose, presumably because each beneficial mutation involved in adaptation to galactose had a much greater fitness effect than each of the deleterious mutations being removed from sexual populations on glucose. This would explain why sex did not result in a similar purging of deleterious mutations from populations maintained on galactose: mutations that increased growth rates on galactose increased rapidly in frequency, overcoming the weaker selection acting upon variation in the load of deleterious mutations.

Our results show that yeast populations become adapted to a novel environment to which they were at first poorly adapted, but do not show that sex accelerates this adaptation. This would be expected if a small number of mutations, each with relatively large effect, were responsible for adaptation. When genotypes from the galactose-adapted asexual populations are crossed with their ancestors, the distribution of growth rates among the progeny is consistent with the action of single loci with major effects (Fig. 3). Although at the end of our experiment the sexual populations appeared no better adapted to galactose than the asexual populations, it is possible that this adaptation did occur earlier in the experiment in the sexual populations than in the asexuals.

So simple an adapative process may not be typical of natural populations, especially over long periods. No experiment can address the sources of selection in the unique historical circumstances of particular organisms. Moreover, our experimental system did not include the environmental heterogeneity or antagonistic coevolutionary interactions that are often required by theories that invoke the effect of sex in bringing together beneficial combinations of genes. Our experiment has, however, demonstrated the operation of a process that has hitherto existed only as a possibility.

\section{Methods}

Construction and propagation of sexual and asexual populations. Haploid mating type a, haploid mating type $\alpha$, diploid, and sexual base populations were composed of yeast strains $\mathrm{yVB} 110, \mathrm{yVB} 114, \mathrm{yVB} 115$, and equal frequencies of yVB110 and yVB114 (ref. 16), respectively. The only genetic variation in our base populations was the presence in $1 \%$ of each population of retrotransposon Ty 3 and a TRP1 or URA 3 marker, introduced into $\mathrm{VVB} 114$ by transformation. We detected no differences between transformant genotypes and yVB114 on either glucose or galactose $(P=0.36$ and 0.39 , respectively, in $t$-tests of growth rates, measured as described below). All media were supplemented with $20 \mathrm{mgl}^{-1}$ uracil and $40 \mathrm{mgl}^{-1}$ tryptophan. Each experimental cycle began with growth for 3 days at room temperature on solid media in Petri plates, followed by transfer to $25 \mathrm{ml}$ liquid medium in a $250-\mathrm{ml}$ flask shaken at
250 r.p.m. Aliquots of $40 \mu \mathrm{l}$ from each asexual population were spread on plates and refrigerated; the sexual populations were transferred to $25 \mathrm{ml}$ presporulation medium ( $2 \%$ potassium acetate, $2 \%$ peptone, $1 \%$ yeast extract) for $24 \mathrm{~h}$ and then sporulation medium ( $1 \%$ potassium acetate, $0.1 \%$ yeast extract, $0.05 \%$ glucose) for $5 \mathrm{~d}$. Unsporulated cells were killed by overnight digestion in $100 \mu \mathrm{g} \mathrm{ml}^{-1}$ zymolyase and $0.2 \% \beta$-mercaptoethanol. Sibling ascospores were dissociated by shaking at 300 r.p.m. for $30 \mathrm{~min}$ in $5 \mathrm{ml} 0.1 \%$ Tween- 20 and $2 \mathrm{ml}$ glass beads ( $0.4-0.6 \mathrm{~mm}$ diameter) and the ascospores were spread on plates. At this stage, the plated asexual populations were returned to room temperature and the subsequent experimental cycle began.

Assays of fitness. The relative fitness of all genotypes tested was determined either by measuring the growth rates of replicate colonies started from single cells of that genotype, or by competition experiments. The choice was determined by the number of genotypes to be scored: for assays such as the overall comparison of population mean fitnesses (Fig. 1), the number of genotypes to be tested precluded the use of the more laborious and timeconsuming competition procedure. To confirm that genotypes with faster colony growth rates were also superior competitors, we did pairwise competition tests on both glucose and galactose for each of 16 pairs of genotypes taken from the final populations. In 24 of 32 cases, the genotype with the faster growth rate on a particular medium increased significantly in frequency on that medium (Fisher exact test, $P=0.0035$ ).

For the overall comparison of population mean fitnesses, an aliquot from each evolved population was diluted in sterile water and thin-spread on agar. From each population, ten colonies were chosen haphazardly and streaked onto both glucose and galactose plates. After $24 \mathrm{~h}$, an aliquot of each genotype was streaked onto one side of a new plate, and from each genotype 8 individual unbudded cells of typical size were picked up and placed on a $1 \mathrm{~cm} \times 1 \mathrm{~cm}$ grid on the plate using a Singer MSM micromanipulator. On each plate, strain $y \mathrm{VB} 114$, treated as above, was included as a control. After three days' growth at room temperature, the diameter of each colony was digitized using a dissecting microscope and SigmaScan software. For each colony a relative size was calculated by subtracting from its diameter the mean diameter of the $\mathrm{yVB1} 14$ colonies on that plate. When the total variance in colony diameter is partitioned into among-genotype and within-genotype components, $89.6 \%$ of the total variance is genotypic.

The genetics of adaptation to galactose. To determine whether adaptation to the galactose medium involved few or many loci, we crossed one genotype from each population maintained on galactose with yVB110 or with yVB114, sporulated the resulting diploids, and dissected the tetrads. The ancestral strains sporulate poorly, and spore viability in these crosses was low. For each tetrad that yielded four colonies, we transferred aliquots of cells from each colony to a fresh plate and used micromanipulation to determine the mean colony size of each haploid genotype after 3 days' growth. The deviation of each genotype from the mean for that tetrad was then calculated, and the logtransformed results plotted as histograms.

Inbreeding depression. Two genotypes were chosen haphazardly from each of two sexual and two asexual populations that evolved on glucose. Each genotype tested was both outcrossed and inbred (selfed). For selfing, the genotypes were transformed with plasmid pGAL-HO, carrying a galactoseinducible copy of the $\mathrm{HO}$ gene ${ }^{17}$. Transformants were grown on galactose to induce mating-type switching and mating, and mated pairs of cells were 
selected visually and isolated by micromanipulation. The diploid status of selfed genotypes was confirmed by observing that they did not mate with tester strains of either mating type. For outcrossing, each asexual genotype was mated with a genotype from a parallel sexual population (for example, genotype 1 from replicate 1 of the sexual glucose population was mated with genotype 1 from replicate 1 of either the mating-type a or mating-type $\alpha$ asexual glucose population, depending on the mating type of the sexual genotype). Mating cultures were spread on glucose plates, and mated pairs of cells were selected by micromanipulation. The fitnesses $(w)$ of diploids produced by selfing and outcrossing were determined by competitions against a diploid produced by crossing yVB110 with a derivative of yVB114 carrying a URA3 genetic marker, and inbreeding depressions were calculated as $w_{\text {outcrossed }}-w_{\text {inbred }} / w_{\text {outcrossed }}$.

Received 23 April; accepted 9 May 1997.

1. Bell, G. The Masterpiece of Nature (University of California, San Francisco, 1982).

2. Maynard Smith, J. The Evolution of Sex (Cambridge Univ. Press, 1978).

3. Kondrashov, A. S. Deleterious mutations and the evolution of sexual reproduction. Nature 336, 435 440 (1988).

4. Charlesworth, D., Morgan, T. M. \& Charlesworth, B. Mutation accumulation in finite outbreeding and inbreeding populations. Genet. Res. 61, 39-56 (1993).

5. Peck, J. R. A ruby in the rubbish: Beneficial mutations, deleterious mutations and the evolution of sex. Genetics 137, 597-606 (1994)

6. Malmberg, R. L. The evolution of epistasis and the advantage of recombination in populations of bacteriophage T4. Genetics 86, 607-621 (1977).

7. McPhee, C. P. \& Robertson, A. The effect of suppressing crossing-over on the response to selection in Drosophila melanogaster. Genet. Res. 16, 1-16 (1970).

8. Golemis, E. A., Gyuris, J. \& Brent, R. in Current Protocols in Molecular Biology (eds Ausubel, F. M. et al.) 13.1-13.14.17 (Wiley, Boston, 1994).

9. Birdsell, J. \& Wills, C. Significant competition advantage conferred by meiosis and syngamy in the yeast Saccharomyces cerevisiae. Proc. Natl Acad. Sci. USA 93, 908-912 (1996).

10. Kunz, B. A. \& Haynes, R. H. in The Molecular Biology of the Yeast Saccharomyces Vol. 1 (eds Strathern, J., Jones, E. W. \& Broach, J.) 371-414 (Cold Spring Harbor, Plainview, 1981).

11. Mortimer, R. K. \& Schild, D. in The Molecular Biology of the Yeast Saccharomyces Vol. 1 (eds Strathern, J., Jones, E. W. \& Broach, J.) 341-370 (Cold Spring Harbor, Plainview, 1981).

12. Menees, T. M. \& Sandmeyer, S. B. Transposition of the yeast retroviruslike element Ty3 is dependent on the cell cycle. Mol. Cell. Biol. 14, 8229-8240 (1994).

13. Muller, H. L. The relation of recombination to mutational advance. Mutat. Res. 1, 2-9 (1964).

14. Crow, J. F. in The Evolution of Sex: an Examination of Current Ideas (eds Michod, R. E. \& Levin, B. R.) 56-73 (Sinauer, Sunderland, MA, 1988)

15. De Visser, J. A. G. M., Hoekstra, R. F. \& Van Den Ende, H. The effect of sex and deleterious mutation on fitness in Chlamydomonas. Proc. R. Soc. Lond. B 263, 193-200 (1996)

16. Bilanchone, V. W. et al. Positive and negative regulatory elements control expression of the yeas retrotransposon Ty3. Genetics 134, 685-670 (1993).

17. Herskowitz, I. \& Jensen, R. E. Putting the $H O$ gene to work: practical uses for mating-type switching Methods Enzymol. 194, 132-146 (1990).

Acknowledgements. We thank S. Sandmeyer for yeast strains and plasmids; H. Bussey for plasmids an the use of his micromanipulator; $\mathrm{H}$. Reiswig for the use of digitizing equipment; D. Schoen for comment on the manuscript; and A. Kondrashov for helpful criticism. This research was supported by NSERC an FACR grants to G.B., NSERC and FCAR scholarships to C.Z., and grants to R. Lenski.

\section{The heritability of IQ}

\section{B. Devlin ${ }^{\star} \nmid$, Michael Daniels $\uparrow$ \& Kathryn Roeder $\dagger$}

* Department of Psychiatry, University of Pittsburgh School of Medicine, Pittsburgh, Pennsylvania 15213, USA

$\dagger$ Department of Statistics, Carnegie Mellon University, Pittsburgh,

Pennsylvania 15213, USA

IQ heritability, the portion of a population's IQ variability attributable to the effects of genes ${ }^{1}$, has been investigated for nearly a century, yet it remains controversial. Covariance between relatives may be due not only to genes, but also to shared environments, and most previous models have assumed different degrees of similarity induced by environments specific to twins, to non-twin siblings (henceforth siblings), and to parents and offspring. We now evaluate an alternative model that replaces these three environments by two maternal womb environments, one for twins and another for siblings, along with a common home environment. Meta-analysis of 212 previous studies shows that our 'maternal-effects' model fits the data better than the 'familyenvironments' model. Maternal effects, often assumed to be negligible, account for $20 \%$ of covariance between twins and $5 \%$ between siblings, and the effects of genes are correspondingly reduced, with two measures of heritability being less than $50 \%$. The shared maternal environment may explain the striking correlation between the IQs of twins, especially those of adult twins that were reared apart. IQ heritability increases during early childhood, but whether it stabilizes thereafter remains unclear. A recent study of octogenarians ${ }^{2}$, for instance, suggests that IQ heritability either remains constant through adolescence and adulthood $^{3}$, or continues to increase with age $^{2}$. Although the latter hypothesis has recently been endorsed ${ }^{4}$, it gathers only modest statistical support in our analysis when compared to the maternal-effects hypothesis. Our analysis suggests that it will be important to understand the basis for these maternal effects if ways in which IQ might be increased are to be identified.

Despite its conceptual simplicity, IQ heritability has engendered vitriolic debates throughout this century, debates that are now recurring ${ }^{5}$ following the publication of The Bell Curve by $\mathrm{R}$. Herrnstein and C. Murray ${ }^{6}$. Part of this controversy arises because IQ heritability is not well characterized. Paradoxically, direct versus indirect analytic methods, both of which attempt to estimate genetic effects on IQ unencumbered by environmental effects, yield markedly different estimates ${ }^{7}$. Direct studies presumably eliminate environmental covariance by evaluating relatives raised apart; indirect studies presumably eliminate environmental covariance by contrasting results from different study designs that have complementary environmental covariance terms. Contrary to their identical objectives, the former typically produces notably larger IQ heritability estimates. We postulate that this paradox is attributable in large part to the presence of unacknowledged maternal effects on IQ.

In traditional quantitative genetic studies, the environment is divided into maternal and external constituents ${ }^{1}$. We expand this dichotomy to take account of the realities of IQ adoption studies, lumping maternal environment and any shared external environment of adopted children into a single 'pre-separation environment'. Because the duration of shared external environment is usually small, pre-separation and maternal effects are roughly equivalent. Both are distinct from maternal inheritance, which is sometimes called a maternal effect ${ }^{8}$.

Our IQ analyses focus on the magnitude of the additive and nonadditive genetic components ${ }^{1}$, estimated to explain $60-85 \%$ of the variation in IQ from adult monozygotic twin studies, and the magnitude of maternal effects, usually assumed to be negligible. To estimate these effects, we analysed 212 IQ studies (or more precisely, correlations) based on 50,470 distinct pairings. The analysis included 204 correlations from studies of zero and firstdegree relatives or their adoptive counterparts ${ }^{9}$. We supplemented this set with some new twin studies published after 1981: a study of monozygotic twins reared apart ${ }^{10}$, the Swedish adoption/twin study of aging of monozygotic twins reared together and apart and dizygotic twins reared together ${ }^{11}$, and two studies of monozygotic and dizygotic adult twins reared together ${ }^{12}$.

Each IQ correlation and related sample size is classified by kind of study (Fig. 1). We evaluate these data using a standard quantitative genetic model for the components of variance (Table 1) and Bayesian meta-analysis ${ }^{13}$, a standard technique for combining information across studies. Our model is built on two levels of distributional assumptions: we assume a likelihood model for the observed correlations among relatives in each type of study; and we specify a prior distribution for the parameters of the model. We assume any standardized component of variance (positive correlation) is a priori equally likely to lie between zero and one. These prior distributions make the Bayesian parameter estimates similar to maximum-likelihood estimates.

All correlations were Fisher-transformed and, after transformation, observed correlations from the same study design follow a normal distribution if they differ only by measurement error. However, several studies are outliers under a normal likelihood, 